\title{
Overexpression of the truncated form of Livin reveals a complex interaction with caspase-3
}

\author{
GUI-HONG LIU ${ }^{1 *}$, CHUN WANG ${ }^{2 *}$ and ZHEN-YU DING ${ }^{1}$ \\ ${ }^{1}$ Division of Thoracic Cancer and ${ }^{2}$ The Department of Endocrinology, West China Hospital, \\ State Key Laboratory of Biotherapy, Sichuan University, Chengdu 610041, P.R. China
}

Received November 25, 2012; Accepted January 21, 2013

DOI: 10.3892/ijo.2013.1883

\begin{abstract}
Disruption in apoptosis are involved in cancer development and progression. Livin- $\beta$, has been identified as a critical modulator for cell death in several tumor cell lines. It was demonstrated that a truncated fragment of Livin- $\beta$ (tLivin) without its N-terminal 52 amino acids is produced in cells through protein cleavage. However, the biological consequence of the cleavage remains largely ignored. In the present study, we report that tLivin exerted a pro-apoptotic effect on cells. The subcellular localization of tLivin was mainly restricted to the cytoplasm. To explore the underlying mechanism, we observed an elevated caspase-3 activity which may account for the apoptosis. Furthermore, we observed that tLivin was further cleaved into a smaller fragment in cells. This second cleavage was possibly related to activated caspase- 3 . The resulted C-terminal fragment (livC) was an anti-apoptotic factor. Our study may help to deepen our understanding of the role of Livin in the regulation of cell death.
\end{abstract}

\section{Introduction}

Disruption in apoptosis is involved in cancer development and progression, where insufficient apoptosis contributes to the process of carcinogenesis and acquirement of therapeutic resistance $(1,2)$. Apoptosis is initiated and executed by proteases known as caspases whose activation is believed to be required for apoptosis to occur in most instances (3), whereas the inhibitor of apoptosis (IAP) family of proteins is believed to inhibit apoptosis as potent caspase inhibitors (4).

Livin- $\beta$, also known as melanoma inhibitor of apoptosis protein (ML-IAP), has been identified as a member of the IAP

Correspondence to: Dr Zhen-Yu Ding, Division of Thoracic Cancer, Cancer Center, West China Hospital, State Key Laboratory of Biotherapy, Sichuan University, Guoxue Lane 37, Chengdu 610041, P.R. China

E-mail: dingzy333@163.com

*Contributed equally

Key words: Livin, apoptosis, caspase, A549 family (5-7). Livin- $\beta$ contains a single BIR (baculoviral IAP repeat) domain as well a carboxyl-terminal RING domain. It was supposed to suppress downstream caspase-3 and -7 and protect cells from proapoptotic stimuli. An alternative splicing variant $\alpha$ isoform was also identified, which is almost identical except for a 54-bp truncation in exon 6 (8). It seems the two isoforms do not differ significantly in their biological activities.

The expression of Livin- $\beta$ was detectable in a panel of tumor types, including melanoma, leukemias, bladder cancer, breast cancer, cervical cancer, colon cancer, nasopharyngeal cancer and different forms of lung cancer (9-12). Our previous study indicated the expression profile of Livin- $\beta$ was restricted to melanoma, but not melanocytic naevus (13). We also found Livin- $\beta$ was hardly detectable in astrocytoma (14). Taken together, these observations implicate Livin- $\beta$ may play a role for the variant malignant phenotype of tumors.

Noteworthy, Livin- $\beta$ undergoes site-specific cleavage in cells by effector caspase- 3 and -7 and a truncated fragment (tLivin) without its N-terminal 52 amino acids was produced (15). However, the biological consequence of the cleavage remains largely ignored. Here, we show ectopic expression of the truncated fragment tLivin was able to induce the activation of caspase- 3 and this fragment was further cleaved into a protective C-terminal fragment in cells.

\section{Materials and methods}

Plasmid construction. The full-length cDNA of Livin- $\beta$ was amplified from total RNA isolated from fresh tissue of a metastatic malignant melanoma by RT-PCR with the following primers: 5'-AAT AGA TCT CAT GGG ACC TAA AGA CAG TGCC-3' (sense) and 5'-AAT GGA TCC GGA CAG GAA GGT GCG-3' (antisense). When the product of the expected size was obtained, it was inserted into UA cloning vector pDrive (Qiagen, Valencia, CA, USA) and then subcloned into pcDNA 3.1 (Invitrogen, Calsbad, CA, USA) to obtain the expression plasmid. The construct myc-tLivin expressing Livin- $\beta$ lack of N-terminal 52 amino acids with myc tag at its N-terminus was generated by cloning the PCR product into pCMV-myc vector (Clontech, Palo Alto, CA, USA). To create an expression plasmid for the full-length ML-IAP with a C-terminal green fluorescence protein (GFP) fusion, PCR amplification was performed with Pfu polymerase (Fermentas, 
St. Leon Rot, Germany) using the UA clone as a template. GFPliv was then generated by cloning the PCR product into the BamHI and XhoI sites of pEGFP-N1 (Clontech). GFP-tLivin and GFPlivC were constructed by the same strategy. To create a vector expressing full-length ML-IAP with an N-terminal hexahistidine tag, the sequence encompassing Livin- $\beta$ was generated by PCR and cloned into the BamHI and HindIII sites of PQE30 (Qiagen). The construct expressing Livin- $\beta$ lacking $\mathrm{N}$-terminal 52 amino acids was made in the same manner. The expression construct for caspase- 3 contained a hexahistidine tag at the $\mathrm{C}$ terminus of the full-length protein was kindly provided by Professor G.S. Salvesen. All the constructs were verified by sequencing.

Site-directed mutagenesis. Site-directed mutagenesis of tLivin was conducted to generate a D220A substitution mutation by over-lap PCR with the following mutagenic primers: 5'-GCG CCT CCA CAG CCC TGG CTC-3' and 5'- AGC CAG GGC TGT GGA GGCG-3', combined with standard primers matching the regions of flanking the coding DNA in expression vector PQEtLivin. The DNA with expected point mutatin was inserted into the TA cloning plasmid pMD18-T (Takara). After the mutant construct was confirmed by sequencing, the DNA was subcloned into PQE30 for expression in bacteria.

Cell culture, transfection and western blotting. Human A549 lung adenocarcinoma cells, human embryonic kidney 293 cells and colon cancer HCT116 cells harboring wild-type P53 were purchased from the American Type Culture Collection. HCT116 cells with P53 knock-out were donated by Professor Vogelstein (Howard Hughes Medical Institute). The cells were maintained in Dulbecco' modified Eagle's medium (Invitrogen) supplemented with $10 \% \mathrm{FBS}$ and antibiotics at $37^{\circ} \mathrm{C}$.

For transfection of cells, a standard lipofectin method was employed. Cells were seeded in 6-well plates or 96-well plates. Lipofectamine 2000 (Invitrogen) was used at a ration of $2.5 \mu \mathrm{g} / \mu \mathrm{l}$ in input plasmid and lipofectin-DNA complexes were incubated with cells for $6 \mathrm{~h}$ at $37^{\circ} \mathrm{C}$.

Forty-eight hours after transfection, cells were harvested in lysis buffer [50 mM Tris-buffered $\mathrm{HCl}, 1 \% \mathrm{NP}-40,1 \%$ SDS, $100 \mathrm{mM}$ EDTA, $100 \mathrm{mM}$ PMSF, 0.5\% Triton X-100 and $1 \mathrm{mM}$ proteinase inhibitor cocktail (Sigma)] and protein were quantified using Bradford protein assay reagent (Bio-Rad). Total proteins were resolved by SDS polyacrylamide (Bio-Rad) gel electrophoresis. Proteins were electroblotted to PVDF membrane (Amersham Biosciences Ltd., Little Chalfont, UK) and then incubated with block solution (5\% non-fat milk, $0.1 \%$ Tween-20, in TBS) at room temperature for $1 \mathrm{~h}$. Following blocking, the membrane was incubated with an appropriate primary antibody and then incubated with a corresponding horseradish peroxidase conjugated or fluorescein isothiocyanate (0 FITC)-tagged secondary antibody. The blots were developed by an enhanced ECL method (Pierce, Englewood Cliffs, NJ, USA). When FITC-tagged secondary antibody was used, the blot was subjected to Tyhoon Trio fluorescence scanner (Amersham Biosciences).

Reagents and antibodies. Cytotoxic drug cisplatin was purchased from Qilu Pharmaceutical Inc. (Shangdong, China).
Stock preparation of the reagents was stored at $-20^{\circ} \mathrm{C}$. The pan-caspase inhibitor z-VAD-fmk (Promega, Madison, WI, USA) was prepared in DMSO at a concentration of $20 \mu \mathrm{M}$. The fluorogenic substrate z-DEVD-AFC and colorimetric substrate z-DEVD-pNA for caspase-3 were obtained from Molecular Probes.

Primary antibodies used in this study included the following: anti-His mAb (Sigma), anti-myc mAb (Clontech), anti-GAPDH mAb (Kangcheng, Shanghai, China), anti-Livin polyclonal antibody (R\&D, Minneapolis, MN, USA), anticaspase-3 (full-length and active fragment) polyclonal antibody (Santa Cruz), anti-XIAP polyclonal antibody (ProteinTech Group Inc., Chicago, IL, USA), anti-caspase-8 polyclonal antibody (Santa Cruz), anti-cIAP2 polyclonal antibody (Santa Cruz) and anti-GFP polyclonal antibody (Novus, St. Louis, MO, USA).

MTT assay. Survival of cells after treatment was quantified by a modified MTT assay (Promega). Briefly, cells were seeded in 96-well tissue culture plate, in 100- $\mu$ l culture medium. Then the cells were either transfected with different plasmids or treated with cisplatin. MTT labeling solution $(15 \mu \mathrm{l})$ was added to each well and the cells were further incubated at $37^{\circ} \mathrm{C}$ for $4 \mathrm{~h}$. Solubilization solution $(100 \mu \mathrm{l})$ was added to each well for incubation overnight. The absorbance was measured at a wavelength of $570 \mathrm{~nm}$ with a microplate reader (Bio-Rad). Data were the average of 5-wells. Untreated cells served as the indicator of $100 \%$ cell viability.

Expression and purification of proteins. For production of recombinant Livin- $\beta$, full-length cDNA of Livin- $\beta$ was cloned into the plasmid PQE30 (Qiagen). The plasmids were introduced into Escherichia coli strain JM109. The His6tagged protein was prepared from the inclusion body upon induction with $1 \mathrm{mM}$ isopropyl-1-thio- $\beta$-D-galactopyranoside (IPTG) at $37^{\circ} \mathrm{C}$ for $4 \mathrm{~h}$. Purified protein was isolated using Ni-chelating column (Amersham Biosciences) on the AKTA chromatography system (Amersham Biosciences) and eluting with an imidazole gradient from $0-200 \mathrm{mM}$ in $50 \mathrm{mM}$ Tris, $300 \mathrm{mM} \mathrm{Na}_{2} \mathrm{HPO}_{4}, \mathrm{pH}$ 8.0. Coomassie blue staining analysis after SDS-PAGE revealed purity of $>90 \%$. Recombinant Livin- $\beta$ protein was renatured by dialyzing against the buffer containing decreased urea concentration. Its biological activity was confirmed in an in vitro recombinant caspase- 3 activity inhibition assay.

Recombinant tLivin protein was generated in Escherichia coli strain XL-1 blue, induced with $1 \mathrm{mM}$ IPTG at $30^{\circ} \mathrm{C}$ for $4 \mathrm{~h}$. Then the bacteria were lysed and soluble fraction was collected and subjected to Nickel column purification. For the tLivin mutant, the protein was expressed in the same way. Also, the mutant protein was purified with Nickel charged magnetic beads (Toyobo, Osaka, Japan) according to the manufacturer's instructions. Expression and purification of active form of caspase-3 were described previously (16). These proteins were quantified by BCA method.

Preparation and purification of anti-Livin- $\beta$ polyclonal antibody. Anti-Livin- $\beta$ sera were raised in rabbits with purified Livin- $\beta$ protein as immunogen. The immunization protocol was as follows: the rabbit was immunized subcutaneously with 
$300 \mu \mathrm{g}$ protein in complete and incomplete Freund's adjuvant 6 times at one-week interval. Antisera titers were monitored by ELISA where the plate was coated with purified protein. The sera were harvested and polyclonal antibody was purified with an affinity blue-gel (Bio-Rad) on the chromatography system. The purified antibody was assayed for its ability to recognize recombinant and endogenous ML-IAP protein in western blot analysis.

DEVDase activity. The assay was performed on a SpectraMAX M5 plate reader coupled with SoftMax software (Molecular Devices, Sunnyvale, CA, USA) operating in the kinetic mode at $37^{\circ} \mathrm{C}$. DEVDase acitivity was determined by using colorimetric pNA substrates (maximal absorbance at $405 \mathrm{~nm}$ ) or fluorogenic AFC substrate (excitation wavelength $400 \mathrm{~nm}$ and emission wavelength $505 \mathrm{~nm}$ ). Assay buffer was $50 \mathrm{mM}$ HEPES, pH 7.4; 100 mM NaCl, 0.1\% Chaps, 10 mM dithiothreitol and $10 \%$ sucrose. Data were recorded every $30 \mathrm{sec}$ for various periods of time as appropriate for each assay.

Apoptosis assay. Cells were transfected with the indicated GFP fusion plasmid to allow quantitation of transfection efficiency. Green (transfected) cells were then scored as either apoptotic or viable according to standard morphological criteria. Apoptotic cells were typically retracted from the substratum, shrunk and exhibited dramatic membrane blebbing along with the formation of numerous apoptotic bodies. A minimum of 300 transfected cells per treatment was evaluated. The images of GFP expression were captured by an Axiovert 200 inverted fluorescence microscope (Zeiss, Oberkochen, Germany).

Preparation of cytosolic fraction. Cytosolic extracts from 293 cells with different treatments were harvested by gentle scraping into phosphate-buffered saline at $4^{\circ} \mathrm{C}$, pelleted by centrifugation and subsequently washed once in the same buffer. The cell pellet was resuspended in HEB (20 mM Pipes, $10 \mathrm{mM} \mathrm{KCl}, 5 \mathrm{mM}$ EDTA, $2 \mathrm{mM} \mathrm{MgCl}_{2}, 1 \mathrm{mM}$ dithiothreitol, $\mathrm{pH} 7.4$ ) at $4^{\circ} \mathrm{C}$ and pelleted by centrifugation at $1,000 \mathrm{xg}$. The cell pellet was then resuspended in an equal volume of HEB and allowed to swell on ice for $30 \mathrm{~min}$. The cells were then cracked by passing through a 24-gauge needle and pelleted by centrifugation at $16,000 \mathrm{x} \mathrm{g}$ for $30 \mathrm{~min}$ and the supernatant (cytosolic extract) recovered. The resultant pellets were collected as nuclear fraction.

Cell cycle analysis. Cells were trypsinized and prepared for cell cycle analysis using the Vindelov protocol with propidium iodine (PI) for DNA staining (17). Cell cycle analysis was performed using a FACscan (Becton-Dickinsson, San Jose, CA, USA). The data were analyzed and calculated by ModFit LT for Mac V3.1 (Verity Software House, Inc., Topsham, ME, USA).

$\gamma H 2 A X$ immunostaining. Cells grown on the slides were fixed in $4 \%$ paraformaldehyde and permeabilized with $0.1 \%$ Triton $\mathrm{X}-100$. Then $\gamma \mathrm{H} 2 \mathrm{AX}$ Ab (Abcam) diluted in Antibody Diluent (Dako) was applied and incubated at $4^{\circ} \mathrm{C}$ overnight. Alexa fluor 488 conjugated anti-mouse $\mathrm{Ab}$ was applied, followed by DAPI incubation. The ProLong Gold AntiFade Reagent was dripped onto the slides. Finally, the fluorescence was observed under a fluorescence microscope (Axiovert 200).

Co-immunoprecipitation. The co-immunoprecipitation was performed with the c-Myc tag co-IP kit (Thermo Scientific, Rockford, IL, USA). Briefly, cell were transfected with myctLivin as described above. Lysates were immobilized with anti-c-Myc agarose overnight at $4^{\circ} \mathrm{C}$. The agarose was washed twice with TBST and then the c-Myc tagged protein was eluted with elution buffer. The c-Myc tagged tLivin was probed with an anti-Livin antibody as described.

Statistical analysis. Statistical analysis was performed by SPSS 13.0 (IBM software). The 2-side Student's t-test for independent samples was used for the analysis of all data. P-values $<0.05$ were considered to be statistically significant.

\section{Results}

Truncated Livin exerted a pro-apoptotic effect. We introduced the expression plasmid for the truncated Livin (tLivin) into the A549 lung adenocarcinoma cells. Two days after transfection, A549 cells treated with tLivin manifested as retracted from the substratum, shrunken and with membrane blebbing, typical features of cell apoptosis (Fig. 1A). We also used the Hoechst 33258 staining to check for apoptosis after treatment with tLivin. Consistently, tLivin induced apoptosis in A549 cells, which was characterized by chromatin condensation and fragmentation (Fig. 1A). To further evaluate the effects of tLivin, an MTT assay was performed to determine cell viability. The treatment reduced the cell viability compared with empty vector in a dose-dependent manner. The higher dose of tLivin plasmid led to less cell viability compared with the same amount of empty vector (Fig. 1B). We also constructed a tLivin and GFP fusion plasmid (GFP-tLivin) and used this plasmid to evaluate the pro-apoptotic efficiency of tLivin. In this assay, ectopic expression of tLivin led to $\sim 30 \%$ of apoptosis while the apoptosis rate of control GFP treated cells was $<10 \%$ (Fig. 1C). Besides, we also observed that the expression of tLivin sensitized A549 cells to cytotoxic chemotherapeutic agent cisplatin, assayed morphologically or by fluorescence staining (Fig. 1A). In addition, we found the synergistic effect of tLivin with cisplatin was more significant with the higher dose of cisplatin than the lower dose (Fig. 1D). The dose of $15 \mathrm{mg} / \mathrm{ml}$ of cisplatin was chosen for the following experiments.

Truncated Livin synergizes with cisplatin through caspase-3 mediated apoptosis. A synergistic effect of tLivin combined with cisplatin on A549 cells was observed and we sought to explore the possible mechanism. The DNA break repair capacity was determined by immunostaining for $\gamma \mathrm{H} 2 \mathrm{AX}$ after cisplatin treatment. No difference was noticed between tLivin-treated or vector-treated cells (Fig. 2A). Next the cell cycle distribution of A549 cells with or without cisplatin treatment was examined by flow cytometry. The cells transfected with tLivin had even less changes in cell cycle distribution after cisplatin treatment although they were more vulnerable to cisplatin (Fig. 2B). Therefore, the synergistic effects of tLivin could not be attributed to the cell cycle re-distribution. 
A
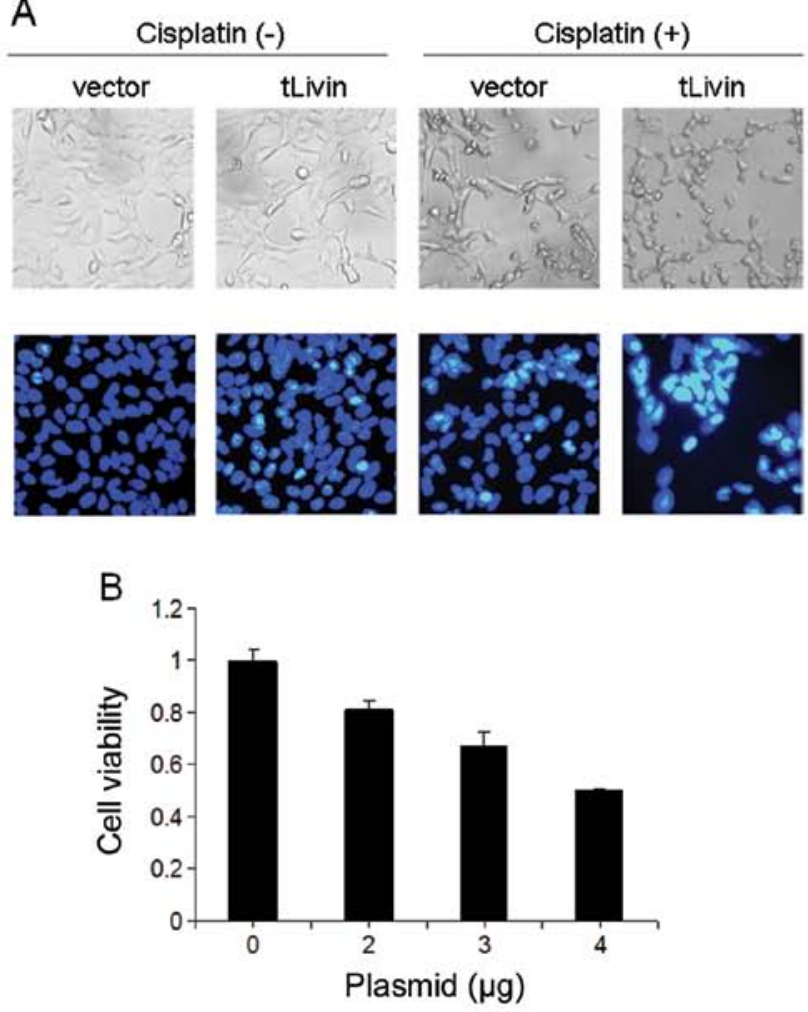

C
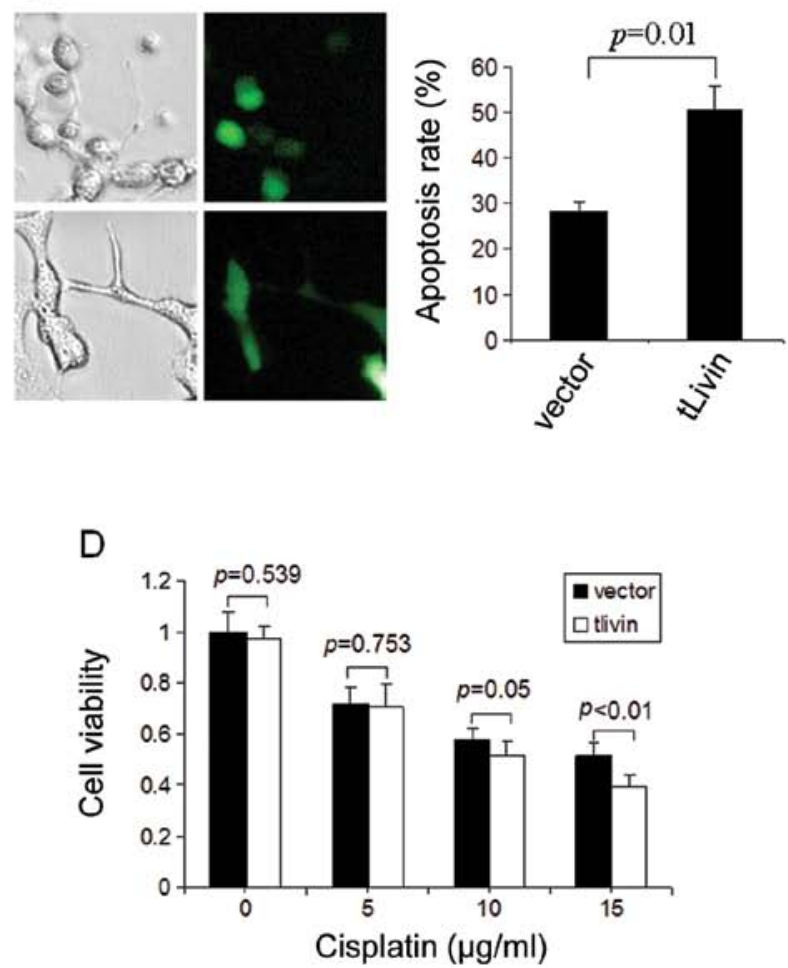

Figure 1. tLivin exerted a pro-apoptotic effect on cells. Cells transfected with myc-tLivin manifested typical morphological changes resembling cell apoptosis in the presence or absence of cisplatin. Hoechst staining also demonstrated higher percentage of apoptosis in cells treated with tLivin, compared with those with empty vector (A). MTT assay showed that cells treated with tLivin had a decreased cell viability (B). Cells transfected with GFP-tLivin were observed under a fluorescence microscope. Apoptosis was scored based on the morphology of cells with GFP signal. Cells treated with GFP-tLivin showed an apoptosis rate $\sim 30 \%$. The data were collected from three independent experiments (C). A549 cells were transfected with the indicated plasmid and then were treated with different concentration of cisplatin. At each concentration of cisplatin, cells transfected with thivin showed a decreased viability compared with those treated with empty vector (D).
The impact of P53 status on the effects of tLivin was also checked. To this end, human colon cancer HCT116 cells with wild-type (HCT116 ${ }^{\mathrm{P53++}+}$ ) or null TP53 $\left(\mathrm{HCT} 116^{\mathrm{P53}-/-}\right)$ were transfected with tLivin and treated with cisplatin. In both cell types, tLivin transfection led to less viability than vector transfection (Fig. 2C).

Then the active form of caspase-3 was examined by Western blotting. The cells transfected with tLivin showed a stronger signal of the $17-\mathrm{kDa}$ form of caspase-3 which indicated a higher activity of caspase-3 in this setting (Fig. 2D). A DEVDase activity assay was further conducted to confirm the observation. In this experiment, the highest DEVDase activity was observed in the tLivin transfected cells, followed by vector transfected ones (Fig. 2E). The cytotoxic effect of tLivin was possibly attributed to apoptosis, therefore an inhibitor of caspase- 3 was used and found to markedly reduce cell death by tLivin or tLivin combined with cisplatin (Fig. 2F).

Truncated Livin was mainly localized in cytoplasm. It was reported that Livin- $\beta$ was localized in the cytoplasm by using an immunostaining method. We wanted to explore the subcellular localization of tLivin. Here, the expression plasmid for GFP-fused tLivin (GFP-tLivin) was constructed to study its distribution in living cells (Fig. 3A). Also the recombinant GFP-Livin- $\beta$ vector was prepared in parallel. We first transfected A549 cells with the constructed plasmid or GFP plasmid. Forty-eight hours later, the living cells were examined by fluorescence microscope. GFP protein displayed an evenly diffused localization throughout the whole cells transfected with pEGFP-N1. GFP-tLivin was mainly found to localize in the cytoplasm (Fig. 3B). Western blot analysis confirmed properly expressed GFP and GFP-tLivin (Fig. 3C). However, the GFP-Livin- $\beta$ vector failed to show any fluorescence with our repeated efforts (data not shown). In Western blotting only fragments of GFP-Livin- $\beta$ fusion protein were detected (Fig. 3C). The exact mechanism underlying the improper expression of GFP-Livin- $\beta$ remains elusive. To further investigate the subcellular localization of tLivin, we carried our subcellular fractionation experiments in Livin or tLivin transfected cells. Livin- $\beta$ was almost completely located in the cytoplasm. In parallel, tLivin was mainly detected in the cytoplasmic fraction (Fig. 3D).

Truncated Livin was cleaved into a smaller fragment in cells. To confirm the expression of tLivin after transfection, lysates prepared from A549 cells transfected with myc-tLivin were immunoblotted with a monoclonal antibody against Myc tag. The 37-kDa myc-tLivin protein band was readily detected in cells transfected with the recombinant expression plasmid. In addition to full-length Myc-tLivin protein, an immunoreactive band of $\sim 22 \mathrm{kDa}$ was also detected after transfection. The additional band was also detected by the antibody against Livin (Fig. 4A), suggesting that thivin may be cleaved after expression. In order to further confirm this observation, similar results were obtained in 293 cells. To exclude the possibility of non-specific immunoreactivity in western blot analysis, the lysates were also probed with a rabbit polyclonal antibody generated against recombinant Livin- $\beta$. Once more, a similar cleavage was observed (Fig. 4B). 
A

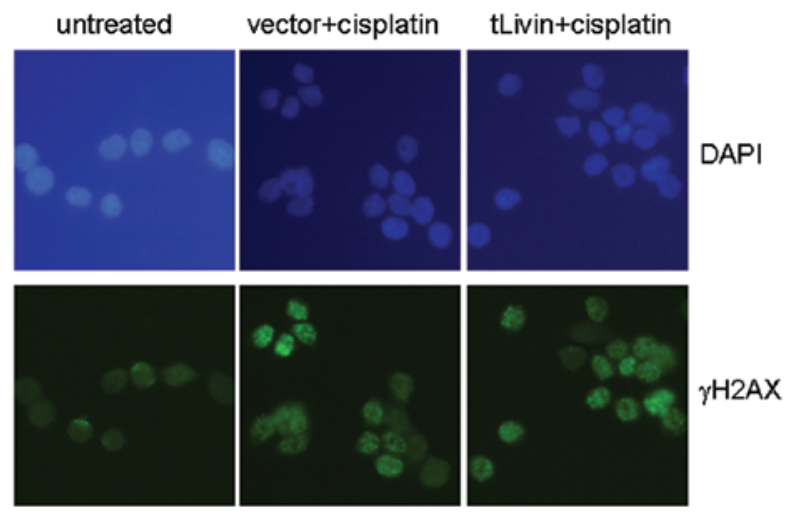

C

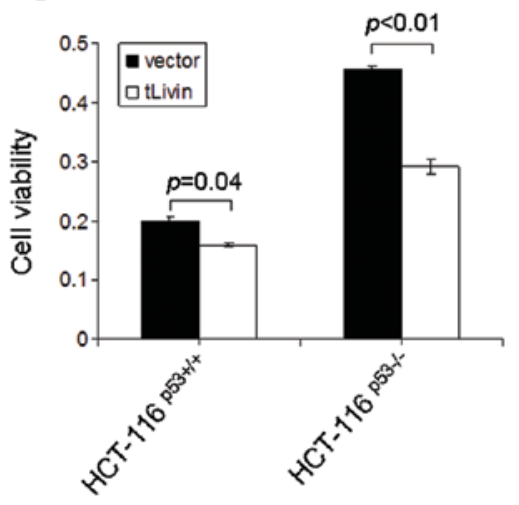

E

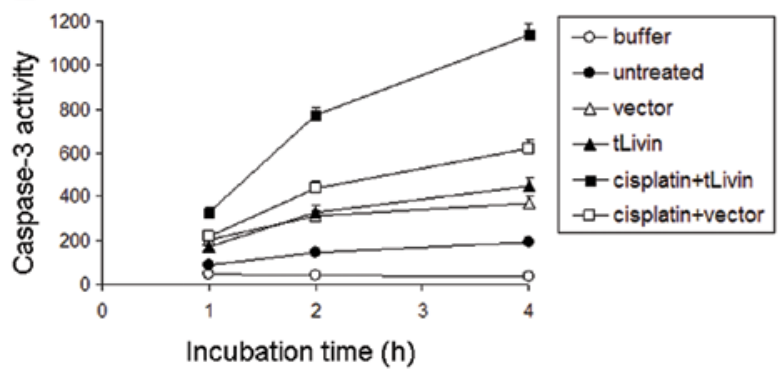

B
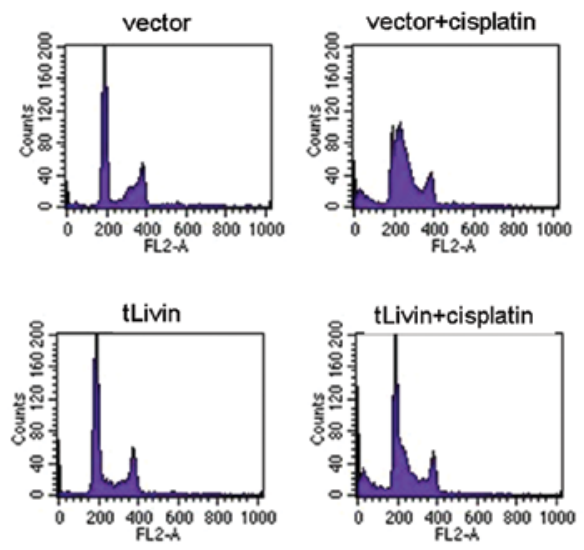

$\mathrm{D}$

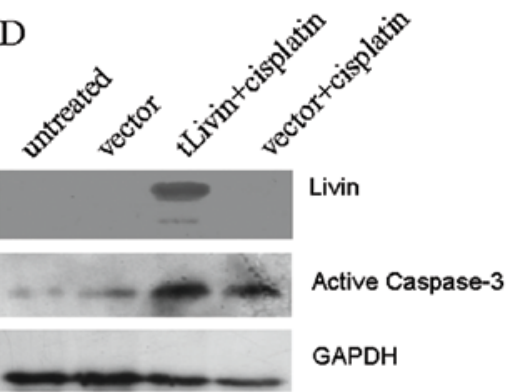

$\mathrm{F}$

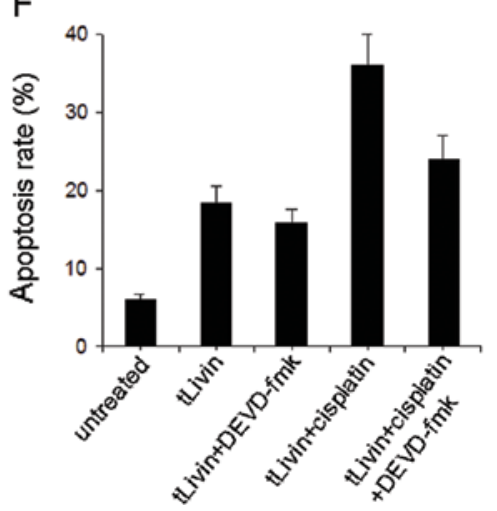

Figure 2. The apoptotic effect of tLivin is mainly attributed to caspase-3 activation. No difference in $\gamma \mathrm{H} 2 \mathrm{AX}$ immunostaining was noticed between tLivintreated or vector-treated cells (A). The A549 cells transfected with tLivin had few changes in cell cycle distribution after cisplatin treatment (B). Human colon cancer HCT116 cells with wild-type or null TP53 were similarly sensitized to cisplatin (C). A549 cells transfected with tLivin showed a stronger signal of the active form of caspase-3 (D). DEVDase assay also confirmed the activation of caspase-3 by tLivin (E). The inhibitor of caspase-3 markedly reduced cell death by tLivin or tLivin combined with cisplatin (F).

Cleavage of tLivin is dependent on caspase-3 activation. We demonstrated that overexpression of tLivin led to apoptosis with activation of caspase- 3 and tLivin mainly localized in the cytoplasm, the same compartment with caspase-3. tLivin was cleaved to smaller fragments. Based on these observations, we wondered whether tLivin cleavage was mediated with caspase-3. To confirm this hypothesis, we used the caspase inhibitor zVAD-fmk to treat A549 cells. We found pre-treatment with zVAD-fmk abrogated the cleavage of tLivin (Fig. 5A). We also performed a co-IP experiment where c-Myc tagged tLivin overexpressed by transfection was precipitated with anti-c-Myc antibody agarose. The precipitated tLivin was confirmed by the anti-Livin antibody, besides the band recognized by anti-caspase- 3 antibody at $\sim 17-\mathrm{kDa}$ size, corresponding to the cleaved caspase-3 fragment (Fig. 5D).

In an attempt to reconstitute the cleavage reaction in vitro, we prepared and purified tLivin recombinant protein from bacteria expression system. The tLivin protein was incubated with pre-activated caspase- 3 protein for $1 \mathrm{~h}$ at $37^{\circ} \mathrm{C}$ and then the reaction mixture was resolved by SDS-PAGE and probed with anti-livin antibody. In this in vitro reaction, tLivin was 

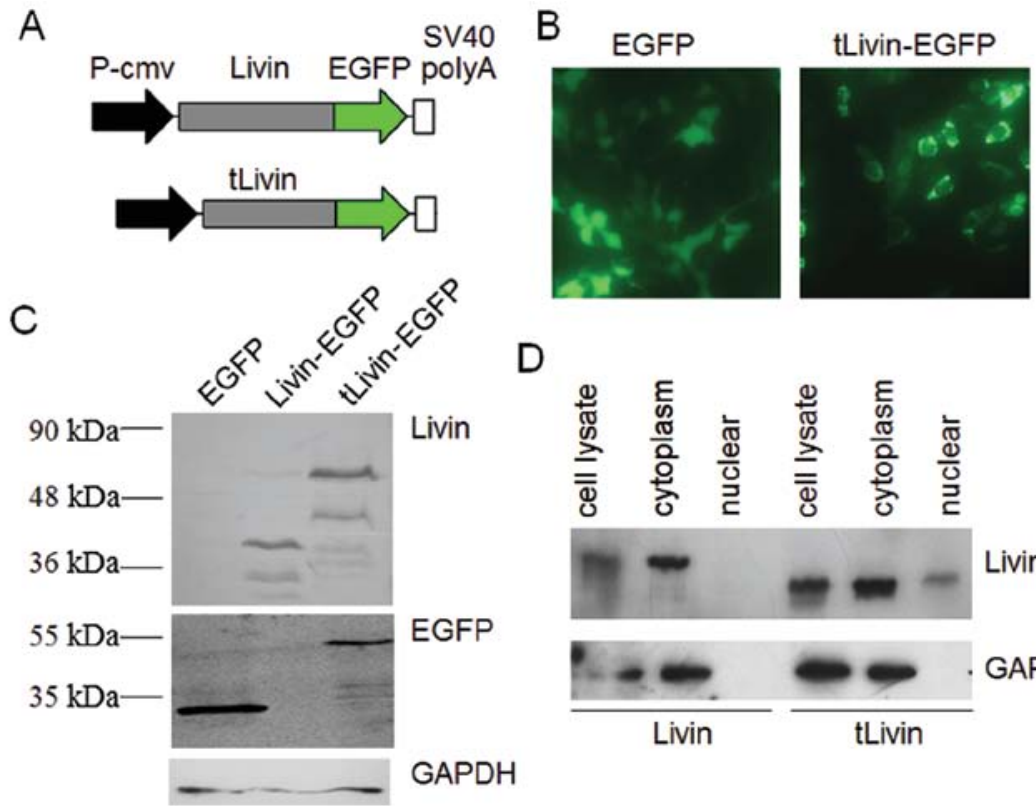

D
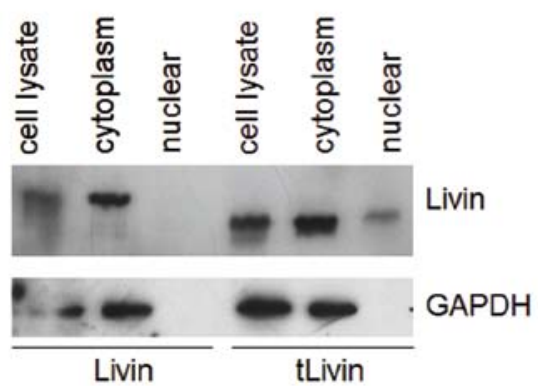

Figure 3. tLivin is mainly localized in the cytoplasm. The expression plasmid for GFP-Livin and GFP-tLivin was constructed as described (A). GFP-tLivin was introduced into cells and the expression of GFP was monitored under a fluorescence microscope. It was mainly localized in the cytoplasm, while GFP alone distributed diffusely in the cells (B). The cell lysates were immunoblotted with anti-GFP and anti-Livin antibodies. The antibody recognized bands of GFP and GFP-Livin of the size predicted (C). Cells overexpressing Livin or tLivin were subjected to subcellular fractionation and tLivin was found to be mainly localized in the cytoplasm fraction in western blot analysis (D).
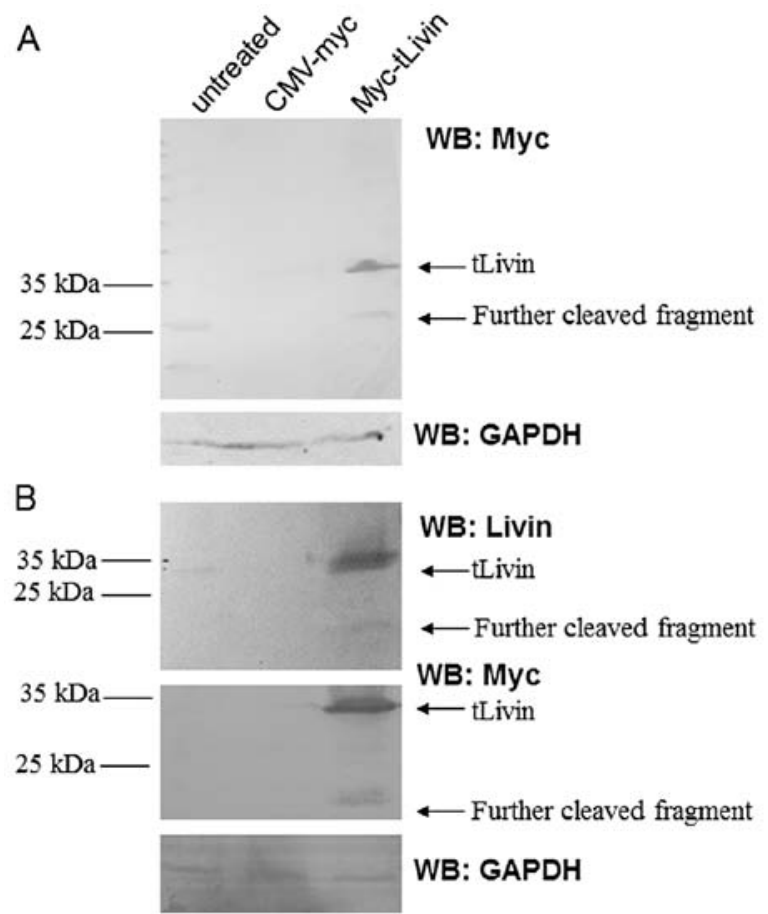

Figure 4. tLivin was cleaved into a smaller fragment. tLivin was ectopically expressed in A549 cells. Then the cells were lysed and lysates were immunoblotted with an anti-myc antibody. We found tLivin was cleaved into a smaller fragment in A549 cells (A) and 293 cells (B). To further confirm this result, the anti-livin antibody was also included to recognize exogenously expressed tLivin protein (B). This, again, confirmed the result. Anti-GAPDH $\mathrm{mAb}$ was used as an internal control.

cleaved into a smaller fragment, similar to that observed in cell lysates (Fig. 5B). However, tLivin protein incubated with a protein control E-cadherin failed to cleave.
Next, the amino sequence of tLivin was scanned and one possible caspase- 3 substrate tetra-peptide (217GARD220) was recognized. To map the cleavage site of tLivin, a sitedirected mutagenesis was performed where the glutid acid in position 220 was mutated to alanine (Fig. 5C). The resulting protein, tLivin D220A, was also incubated with active form of caspase-3. However, this point mutation abrogated the cleavage potential of tLivin (Fig. 5A).

The cleaved C-terminal fragment of tLivin was an antiapoptotic factor. Because the RING finger of XIAP mediated ubiquitination of caspases, we hypothesized the C-terminus of tLivin had anti-apoptotic function. To confirm this hypothesis, we transfected A549 cells with a construct expressing the C-terminus of tLivin (amino acids 221-280, livC) fused with GFP and found it could antagonize the apoptotic effect of cisplatin (Fig. 6B). We also observed a significant decrease in the expression intensity of GFP, which was fused to the $\mathrm{N}$-terminus of livC (Fig. 6A). To further explore the possible mechanism for this effect of livC, the cell lysates were probed with a panel of antibodies. We found the protein content of caspase-3 decreased in cells transfected with livC, both of fulllength and cleaved active form of caspase-3 (Fig. 6C). These data may help to explain the anti-apoptotic effect of livC in cells.

\section{Discussion}

Key mediators in apoptosis regulation are of intense biological interest. Livin- $\beta$ underwent site-specific cleavage in cells upon apoptosis stimulation and a truncated fragment (tLivin) without its $\mathrm{N}$-terminal 52 amino acids was produced (15). In the present study, we constructed an expression plasmid for the truncated fragment of Livin- $\beta$ (tLivin) and validated its pro- 


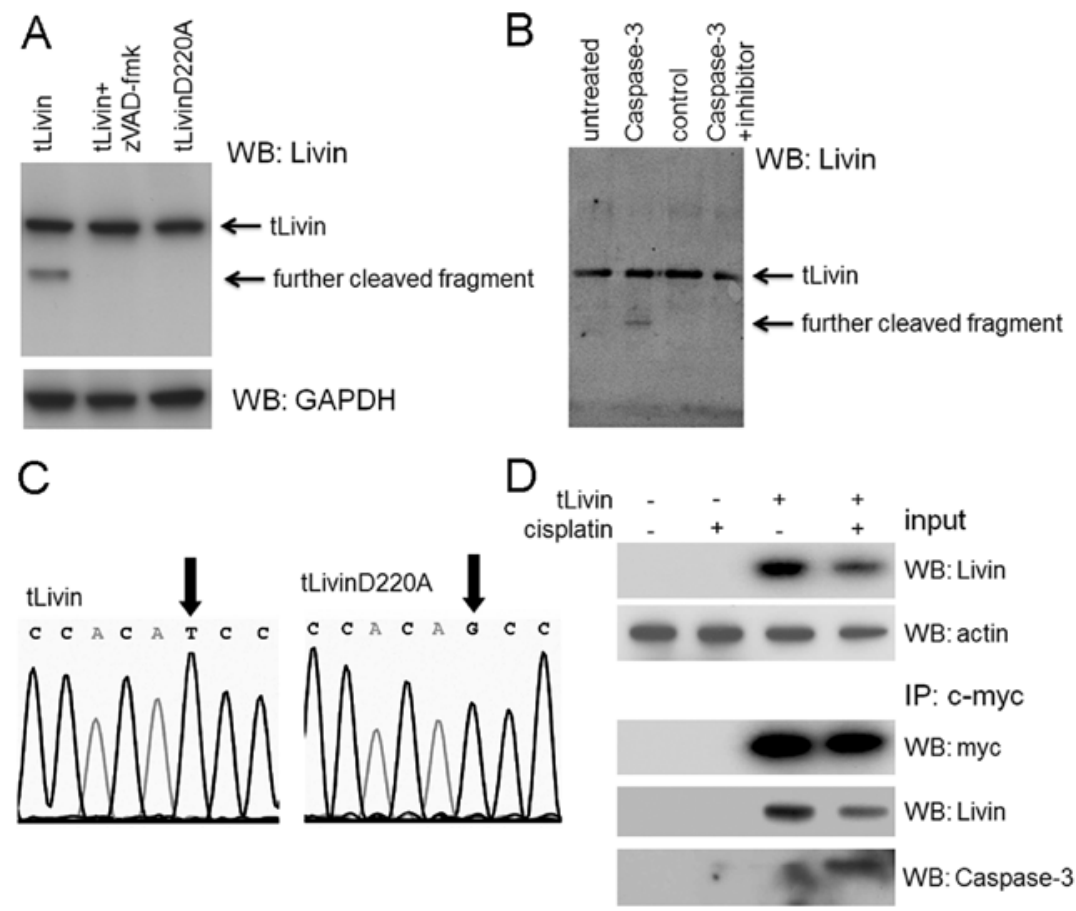

Figure 5. Cleavage of tLivin is caspase-3-dependent. Cells transfected with tLivin were treated with or without zVAD-fmk and the cells were lysed for immunoblotting. Pre-treatment with zVAD-fmk abrogated the cleavage of tLivin in vivo. The site-directed mutant tLivinD220A failed to cleave (A). Recombinant tLivin protein was incubated with active caspase-3 protein and the mixture was resolved on a gel. The resulting cleaved fragment was detected with an antilivin antibody. In contrast, tLivin protein reacted with E-cadherin had no such effect (B). A point mutation of D220A was introduced into tLivin protein (C). An IP assay was performed and confirmed the combination of tLivin with active caspase-3 (D).

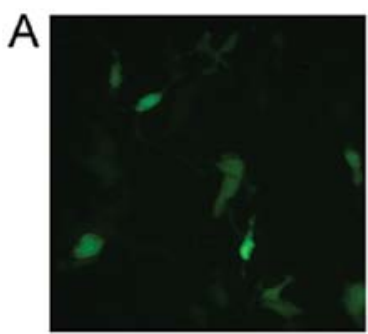

B

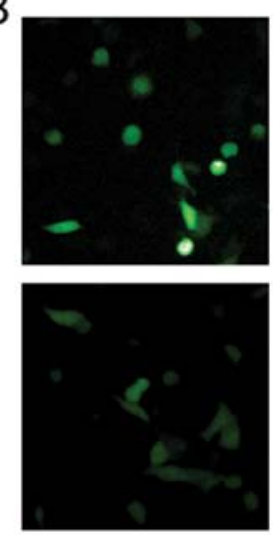

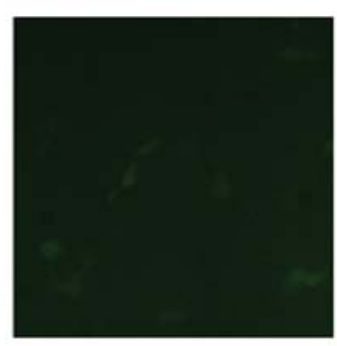

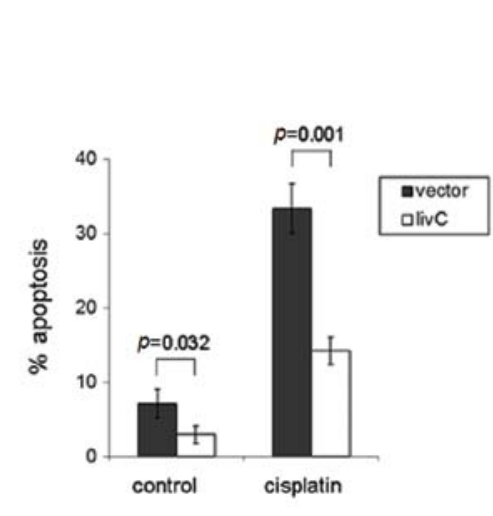

C
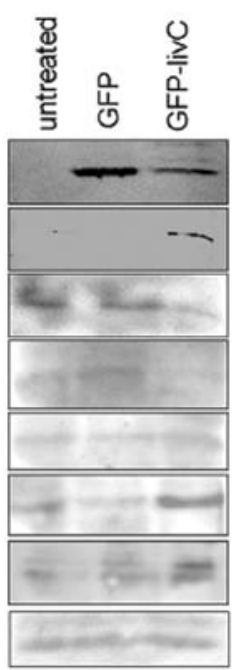

GFP

Livin

Caspase-3 precursor

Caspase-3 fragment

XIAP

C-IAP2

Caspase-8

$\beta$-actin

Figure 6. livC is an anti-apoptotic factor. A plasmid for the cleaved C-terminal fragment of tLivin (livC) was constructed, where livC was fused to GFP as a carrier. The expression of GFP-livC was much weaker than GFP alone under a fluorescence microscope (A). Cells were treated with cisplatin and the apoptosis was counted. Cells overexpressing livC showed less apoptosis. Also the apoptosis rate was almost $20 \%$ less than the control (B). Cells with different treatment were lysed and a panel of immunoblots was used. In this assay, a decreased content of caspase- 3 was observed (C).

apoptotic effect in lung adenocarcinoma cells. Interestingly, we observed tLivin was further cleaved into a smaller fragment in cells and the cleavage was supposed to be mediated by caspases. Accordingly, the treatment with the caspase inhibitor or introduction of a site-directed mutagenesis of 220Asp to 220Ala abrogated the cleavage. Further, the cleavage was 
reconstituted in vitro with the purified recombinant tLivin protein and active caspase- 3 protein, a reaction that was abrogated by zVAD-fmk. We further constructed the expression plasmid for the cleaved C-terminal fragment (livC) and found it exerted an anti-apoptotic effect.

The first 52 amino acids was removed from Livin- $\beta$ and a truncated form (tLivin) with pro-apoptotic effects was produced thereafter (15). The cleavage was proposed to be mediated by caspase-3 or another non-canonical caspase (18). Our study observed the pro-apoptotic fragment tLivin activates caspases and is cleaved into even smaller fragments by the latter. The $\mathrm{C}$-terminal fragment produced by the second cleavage was with anti-apoptotic activity which was possibly attributed to its ubiquitination activity. Cells may use this negative feed-back mechanism to avoid the magnification of cell death signal. Thus, our study reveals a complex interaction between Livin- $\beta$ and caspases and helps to dissect the full functional spectrum of Livin- $\beta$ in cells.

Nachimias et al (15) reported that the Livin $\beta$ was cleaved in vivo by caspase-3, however, in their experiments the sitedirected mutagenesis of D220A failed to abrogate the cleavage. In our study the mutant of tLivinD220A lost the potential of being cleaved by active form of caspase-3. This difference, we thought, was due to the different recognition efficiency of substrate sequence. In fact, the optimal sequence for caspase-3 recognition was DEVD (19). The sequence neighboring amino acid 52 of Livin- $\beta$ was DVHD, which was similar to the original sequence to a greater extent than that of neighboring amino acid 220, GARD. This possibly explained the observed cleavage in our experiment where the optimal sequence had been removed.

We found the C-terminus of Livin- $\beta$, containing the RING motif, exerted anti-apoptotic effects on cells. Similarly, the function of RING motif has been discussed for other members of IAP family. Suzuki et al reported the RING motif in XIAP promotes proteasomal degradation of caspase- 3 and enhances the anti-apoptotic effect of XIAP (20). On the contrary, Silke et al failed to observe such an effect (21). In their study, the RING motif in XIAP mediated the degradation of other IAPs and actually promoted cell death. From these reports and others (22), one may speculate the RING motif probably have variant impact on cells, depending on different cell type and stimuli. Our results were supported by a report that RING motif in Livin- $\beta$ enhanced cell survival and this was possibly through the degradation of Smac/DIABLO (23).

Livin- $\beta$ was reported to exert anti-apoptotic effects against detrimental stimuli (5-7), however controversies remain on the mechanism underlying its anti-apoptotic activity. It was initially reported to suppress downstream effector caspase-3 and-7 through its BIR domain (5-7). However, later experiments failed to prove this notion. Vucic et al reported Livin merely weakly inhibited caspase- 9 activity with inhibition constant $K i \sim 3-5 \mu \mathrm{M}$. Also, it was argued that Livin might regulate apoptosis by sequestering SMAC from XIAP (24). Another report awaiting for further confirmation stated that inhibition of apoptosis by Livin might involve the TAK1/ JNK1 pathway (25). In our study, we found that the C-terminal fragment potently inhibits apoptosis induced by cisplatin. We propose it was attributed to the decreased expression level of caspase- 3 which was possibly related to the ubiquitination property of the RING domain, probably the anti-apoptotic effect of Livin- $\beta$ was also partially attributed to the RING domain.

Previous reports indicated Livin- $\beta$ was a cytoplasmic protein and tLivin was also localized in the cytoplasm fraction $(6,26)$. These reports were in good agreement with the present study. Interestingly, we also found the fragment containing only the RING distributed diffusely throughout the whole cell. The findings argue for the region flanking of the BIR domain to be critical for proper subcellular localization of ML-IAP and tLivin. Although lacking of data to demonstrate the relationship between the subcellular localization and the different biological properties of fragments of Livin- $\beta$, similar studies have been proposed for other key molecules in apoptosis regulation. For example, Smac/DIABLO located in the mitochondria and was released into the cytoplasm during apoptosis (27). The different nuclear and cytoplasmic localization pattern of Survivin determined its different action in apoptosis (14). We might reason that the proper localization of Livin- $\beta$ was important for its function in vivo.

Livin- $\beta$ has been proposed as critical for therapeutic resistance in tumors (28). In addition, the prognostic value of Livin- $\beta$ was examined in tumor samples retrospectively at the mRNA or protein level (9-12). The Livin- $\beta$ expression was associated with poor prognosis at least in lung, bladder and nasopharyngeal cancer. Efforts were devoted to interfere with the activity of Livin- $\beta$ in order to improve the prognosis of cancer patients with Livin- $\beta$ expression. siRNA or small peptide were developed capable of efficiently disrupting the function of Livin- $\beta$ in cells, rendering cells vulnerable to treatment $(29,30)$. Cancer gene therapy and cancer vaccine based on Livin- $\beta$ were also tested in preclinical models $(31,32)$. All these studies highlight the value of Livin- $\beta$ as a therapeutic target. Because tLivin has pro-apoptotic effects, its potential therapeutic usage has been proposed (33). However, the present study provides a deeper insight into this scenario. We suggest the C-terminal fragment of tLivin has protective effects on cells and impedes the therapeutic benefits. More efforts are needed to fulfill the maximum therapeutic potential of tLivin.

In conclusion, the present study focused on the fragment of Livin- $\beta$ without its $\mathrm{N}$-terminal 52 amino acids tLivin. It was shown that tLivin localized in the cytoplasm and it was further cleaved by caspase- 3 to an anti-apoptotic factor. Our study may contribute to the elucidation of functional spectrum of Livin- $\beta$.

\section{Acknowledgements}

The authors thank Professor G.S. Salvesen for providing the expression plasmid for caspase-3 and Professor B. Vogelstein for the HCT-116p53-- cells. This study was supported by grants from National Natural Science Fund of China (81272684, 30901756) and Doctoral Fund of Ministry of Education of China (20090181120100).

\section{References}

1. Ghobrial IM, Witzig TE and Adjei AA: Targeting apoptosis pathways in cancer therapy. CA Cancer J Clin 55: 178-194, 2005. 
2. Zhivotovsky B and Orrenius S: Carcinogenesis and apoptosis: paradigms and paradoxes. Carcinogenesis 27: 1939-1945, 2006.

3. Taylor RC, Cullen SP and Martin SJ: Apoptosis: controlled demolition at the cellular level. Nat Rev Mol Cell Biol 9: 231-241, 2008.

4. Srinivasula SM and Ashwell JD. IAPs: what's in a name? Mol Cell 30: 123-135, 2008

5. Kasof GM and Gomes BC: Livin, a novel inhibitor of apoptosis protein family member. J Biol Chem 276: 3238-3246, 2001.

6. Vucic D, Stennicke HR, Pisabarro MT, et al: ML-IAP, a novel inhibitor of apoptosis that is preferentially expressed in human melanomas. Curr Biol 10: 1359-1366, 2000

7. Lin JH, Deng G, Huang Q and Morser J: KIAP, a novel member of the inhibitor of apoptosis protein family. Biochem Biophys Res Commun 279: 820-831, 2000.

8. Ashhab Y, Alian A, Polliack A, et al: Two splicing variants of a new inhibitor of apoptosis gene with different biological properties and tissue distribution pattern. FEBS Lett 495: 56-60, 2001.

9. Tanabe H, Yagihashi A, Tsuji N, et al: Expression of survivin mRNA and livin mRNA in non-small-cell lung cancer. Lung Cancer 46: 299-304, 2004

10. Gazzaniga P, Gradilone A, Giuliani L, et al: Expression and prognostic significance of Livin, Survivin and other apoptosisrelated genes in the progression of superficial bladder cancer. Ann Oncol 14: 85-90, 2003.

11. Choi J, Hwang YK, Sung KW, et al: Expression of Livin, an antiapoptotic protein, is an independent favorable prognostic factor in childhood acute lymphoblastic leukemia. Blood 109: 471-47, 2007.

12. Xiang Y, Yao H, Wang S, et al: Prognostic value of Survivin and Livin in nasopharyngeal carcinoma. Laryngoscope 116: 126-130, 2006.

13. Gong J, Chen N, Zhou Q, et al: Melanoma inhibitor of apoptosis protein is expressed differentially in melanoma and melanocytic naevus, but similarly in primary and metastatic melanomas. J Clin Pathol 58: 1081-1085, 2005.

14. Liu X, Chen N, Wang X, et al: Apoptosis and proliferation markers in diffusely infiltrating astrocytomas: profiling of 17 molecules. J Neuropathol Exp Neurol 65: 905-913, 2006.

15. Nachmias B, Ashhab Y, Bucholtz V, et al: Caspase-mediated cleavage converts Livin from an antiapoptotic to a proapoptotic factor: implications for drug-resistant melanoma. Cancer Res 63 : 6340-6349, 2003.

16. Zhou Q, Krebs JF, Snipas SJ, et al: Interaction of the baculovirus anti-apoptotic protein p35 with caspases. Specificity, kinetics and characterization of the caspase/p35 complex. Biochemistry 37 10757-1065, 1998

17. Vindel $\varnothing v$ LL, Christensen IJ and Nissen N: Standardization of high-resolution flow cytometric DNA analysis by the simultaneous use of chicken and trout red blood cells as internal reference standards. Cytometry 3: 328-331, 1983.

18. Yan H, Brouha B, Liu T, et al: Proteolytic cleavage of Livin (ML-IAP) in apoptotic melanoma cells potentially mediated by a non-canonical caspase. J Dermatol Sci 43: 189-200, 2006
19. Timmer JC and Salvesen GS: Caspase substrates. Cell Death Differ 14: 66-72, 2007

20. Suzuki Y, Nakabayashi Y and Takahashi R: Ubiquitin-protein ligase activity of $\mathrm{X}$-linked inhibitor of apoptosis protein promotes proteasomal degradation of caspase- 3 and enhances its anti-apoptotic effect in Fas-induced cell death. Proc Natl Acad Sci USA 98: 8662-8667, 2001.

21. Silke J, Kratina T, Chu D, et al: Determination of cell survival by RING-mediated regulation of inhibitor of apoptosis (IAP) protein abundance. Proc Natl Acad Sci USA 102: 16182-16187, 2005.

22. Huang H, Joazeiro CA, Bonfoco E, et al: The inhibitor of apoptosis, cIAP2, functions as a ubiquitin-protein ligase and promotes in vitro monoubiquitination of caspases 3 and 7. J Biol Chem 275: 26661-26664, 2000.

23. Ma L, Huang Y, Song Z, et al: Livin promotes Smac/DIABLO degradation by ubiquitin-proteasome pathway. Cell Death Differ 13: 2079-2088, 2006.

24. Vucic D, Franklin MC, Wallweber HJ, et al: Engineering ML-IAP to produce an extraordinarily potent caspase 9 inhibitor: implications for Smac-dependent anti-apoptotic activity of ML-IAP Biochem J 385: 11-20, 2005.

25. Sanna MG, da Silva Correia J, Ducrey O, et al: IAP suppression of apoptosis involves distinct mechanisms: the TAK1/JNK1 signaling cascade and caspase inhibition. Mol Cell Biol 22: 1754-1766, 2002.

26. Nachmias B, Lazar I, Elmalech M, et al: Subcellular localization determines the delicate balance between the anti- and proapoptotic activity of Livin. Apoptosis 12: 1129-1142, 2007.

27. Yang QH and Du C: Smac/DIABLO selectively reduces the levels of c-IAP1 and c-IAP2 but not that of XIAP and livin in HeLa cells. J Biol Chem 279: 16963-16970, 2004.

28. Crnkovic-Mertens I, Muley T, Meister M, et al: The antiapoptotic livin gene is an important determinant for the apoptotic resistance of non-small cell lung cancer cells. Lung Cancer 54: 135-142, 2006

29. Crnkovic-Mertens I, Bulkescher J, Mensger C, et al: Isolation of peptides blocking the function of anti-apoptotic Livin protein. Cell Mol Life Sci 67: 1895-1905, 2010.

30. Crnkovic-Mertens I, Hoppe-Seyler F and Butz K: Induction of apoptosis in tumor cells by siRNA-mediated silencing of the livin/ML-IAP/KIAP gene. Oncogene 22: 8330-8336, 2003.

31. Wang R, Lin F, Wang X, et al: Silencing Livin gene expression to inhibit proliferation and enhance chemosensitivity in tumor cells. Cancer Gene Ther 15: 402-412, 2008

32. Xie J, Xiong L, Tao X, et al: Antitumor effects of murine bone marrow-derived dendritic cells infected with xenogeneic livin alpha recombinant adenoviral vectors against Lewis lung carcinoma. Lung Cancer 68: 338-345, 2010.

33. Wang L, Zhang Q, Liu B, et al: Challenge and promise: roles for Livin in progression and therapy of cancer. Mol Cancer Ther 7: 3661-3669, 2008 